\title{
Small-Angle Grain Boundaries in Quasicrystals
}

\author{
Yashodhan Hatwalne, H. R. Krishnamurthy, Rahul Pandit, and Sriram Ramaswamy \\ Department of Physics, Indian Institute of Science, Bangalore 560 012, India
}

\begin{abstract}
The Read-Shockley treatment of small-angle grain boundaries in crystals is generalized to the case of quasicrystals. The dependence of the grain-boundary energy on the angle of mismatch between abutting quasicrystalline grains is calculated. It is found that, even for a symmetric tilt boundary in a quasicrystal, dislocations with at least two types of Burgers vectors are required; these dislocations have to be arranged quasiperiodically along the boundary. The possible clumping of these dislocations to form composites is discussed. Explicit calculations are presented for a pentagonal quasicrystal.
\end{abstract}

Translationally ordered solids, either crystals or quasicrystals, can have grain boundaries, which are interfaces between regions with different orientations. Such boundaries are well known in periodic crystals, ${ }^{1}$ and they have been reported recently in quasicrystals. ${ }^{2}$ Frank $^{3}$ and Read and Shockley ${ }^{4}$ have shown how small-angle grain boundaries in crystals can be described as arrays of dislocations [planar (linear) arrays in three (two) dimensions]. Read and Shockley use this description to calculate the dependence of the (zero-temperature) grainboundary energy on the angle of mismatch between abutting crystalline grains. In this paper we generalize the Read-Shockley treatment of small-angle grain boundaries to quasicrystals.

Our principal results are as follows: (1) Whereas a symmetric tilt boundary (the simplest) in a crystal can be obtained by using an array of only one type of dislocation, ${ }^{4}$ in a quasicrystal it can be obtained only by using an array of at least two types of dislocations, alternating quasiperiodically along the boundary. The dislocations in the array must be chosen and arranged in such a way that the sum of their Burgers vectors has a phonon part that scales as $L$ and a phason part that vanishes as $L^{-1}$, where $L$ is the linear size of the array. This was shown first for an incommensurate smectic liquid crystal. ${ }^{5}$ (2) An array of dislocations that does not satisfy the above conditions leads to strains in the quasicrystals that do not vanish infinitely far from the array. Thus, the energy

$$
E_{\mathrm{el}}-\frac{1}{2} \lambda u_{i i} u_{i i}+\mu u_{i j} u_{i j}+\frac{1}{2} K_{1} w_{i j} w_{i j}+K_{2}\left(w_{x x} w_{y y}-w_{x y} w_{y x}\right)+K_{3}\left[\left(u_{x x}-u_{y y}\right)\left(w_{x x}+w_{y y}\right)+2 u_{x y}\left(w,-w_{, . . .}\right)\right] .
$$

where $u_{i j}=\frac{\dot{2}}{2}{ }_{i} u_{j}+$ fly $\left.\ll_{,-}\right)$is the strain tensor, $w_{i j}$ $=\partial_{i} w_{j}$, and we sum over repeated $i$ and $j$ indices. Note that $E_{\mathrm{el}}$ has rotational invariance built into it: Fields with constant, finite $\boldsymbol{\nabla} \times \mathbf{u}$ do not cost any energy. However, $w$ fields with constant, finite $\boldsymbol{\nabla} \times \mathbf{w}$ do cost energy, because they lead to relative rotations of the density waves (see below) of which the quasicrystal is constituted.

It is easy to see now why in a quasicrystal an array of only one type of dislocation yields an energy proportional to $L^{d}$ : As in a crystal, such an array leads to a relative rotation of the regions of quasicrystal on either side of cost of such an array scales as $L^{d}$ for a $d$-dimensional quasicrystal and not as $L^{d-1}$ as required for a true grain boundary. (3) As in a crystal, ${ }^{4}$ the intensive energy per unit area (length if $d=2$ ) of a grain boundary is $E=C_{1} \theta$ $-C_{2} \theta \ln \theta$, where 9 is the tilt angle, and $C_{1}$ and $C_{2}$ depend on the orientation of the grain boundary and on the elastic constants of the quasicrystal. We calculate these for a symmetric tilt boundary in a pentagonal quasicrystal, ${ }^{6}$ by assuming a specific arrangement of dislocations (see below). (4) We show that, if the phason elastic constants are sufficiently large, dislocations in the grain boundary clump to form composites whose size scales as $(K / \mu)^{1 / 4}$, where $K$ and $\mu$ are typical phason and phonon elastic constants, respectively. (5) We obtain the structure of such a grain boundary (Fig. 1) in a pentagonal quasicrystal by using elastic theory.

In the remaining part of this paper we give the arguments that lead to the results summarized above. These arguments hold for all quasicrystals with dimensions $d \geq 2$. We substantiate these arguments with explicit calculations for two-dimensional, pentagonal quasicrystals.

The elastic energy density of a quasicrystal depends on derivatives of two fields: $u$ (the phonon part) and $w$ (the phason part). [Spatially uniform $u$ (i.e., uniform translations) and spatially uniform w (this shifts density waves relative to one another in a density-wave description) do not cost any energy.] For example, for pentagonal quasicrystals, the elastic energy density is ${ }^{7,8}$ the array; i.e., far from the array there is a $u$ field with uniform nonzero $\mathrm{Vxu}$, which costs no energy. In a quasicrystal, however, energy dislocation has both $u$ and $w$ parts, and so the same argument yields a constant, nonzero $\boldsymbol{\nabla} \times \mathbf{w}$ far from the array. Such a field has a nonzero energy density at infinity. Thus the total energy of an array of only one type of dislocation scales as $L^{d}$ in a quasicrystal. Such an array is clearly not a grain boundary.

We now show how, using more than one type of dislocation, it is possible to construct an array which leads to 


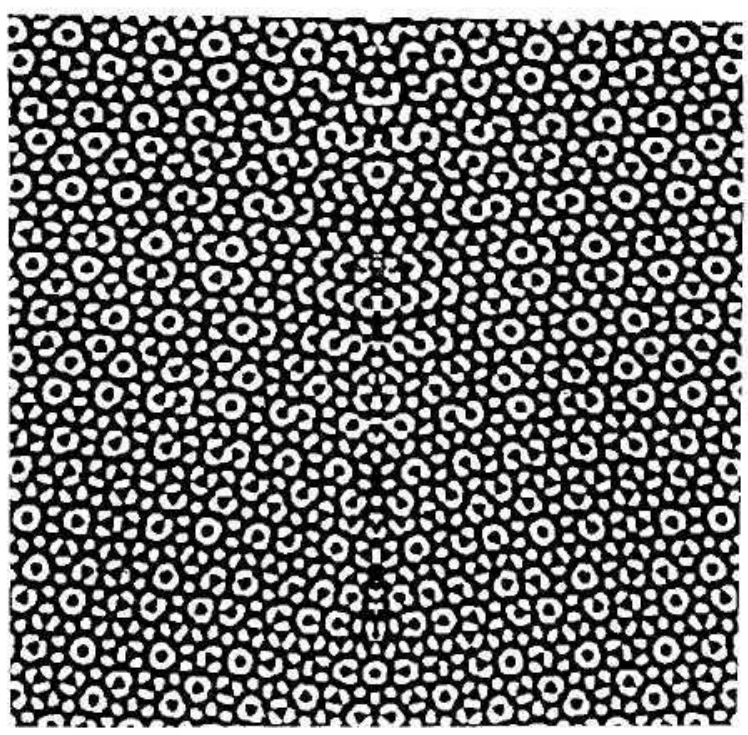

FIG. 1. A pentagonal quasicrystal with a $24.8^{\circ}$ symmetric tilt boundary running vertically down the middle of the picture. The grain boundary was generated by an array of type (2) described in the text.

orientational mismatch between two quasicrystalline regions and has an energy proportional to $L^{d-1}$. Such an array is a natural candidate for a grain boundary. The construction is simple. The dislocations in the array are chosen and arranged such that the sum of their Burgers vectors has a $u$ part proportional to $L$ and a $w$ part that vanishes as $L^{-1}$. The $u$ part produces the desired relative rotation between the regions on either side of the array. Since the $w$ part vanishes as $L^{-1}, \boldsymbol{\nabla} \times \mathbf{w}=0$ far from the boundary. Thus the only contribution to the energy of the array must come from the nonuniform $u$ and $w$ fields in the vicinity of the array. Therefore the energy must scale as $L$ !.

We illustrate below this construction of a grain boundary for a two-dimensional, pentagonal quasicrystal. Dislocations $^{7(b)}$ in such a quasicrystal have four-dimensional Burgers vectors $\mathbf{B}=\mathbf{b} \oplus \mathbf{d}$, where the two-dimensional vectors $b$ and $d$ are

$$
\mathbf{b}=\oint_{d \mathbf{u},} \mathbf{d}=\oint_{d \mathbf{~} \mathbf{w}}
$$

$\mathrm{u}$ and $\mathrm{w}$ are, respectively, phonon and phason fields and the line integrals are along any closed contour that surrounds the core of the dislocation. The four-dimensional hypercubic lattice of these Burgers vectors can be generated by any four of the five vectors $\mathbf{B}_{n}=\left(\mathbf{b}_{n}, \mathbf{d}_{n}\right)$, $\left.n=0-4, \quad \mathbf{b}_{n}=b(\sin (2 n \pi / 5), \cos (2 n \pi / 5)), \quad \mathbf{d}_{n}=\mathbf{b}_{\langle 3 n\rangle}\right\rangle$, and $\langle 3 n\rangle=3 n(\bmod 5)$; no Burgers vector can lie in the $b=0$ or $\mathbf{d}=0$ planes. ${ }^{7(b)}$ To construct a grain boundary, we must, therefore, form an array with more than one type of dislocation such that their w fields cancel in the mean. This is easy to do for a pentagonal quasicrystal: Since $\mathbf{b}_{1}+\mathbf{b}_{4}=2 \mathbf{b}_{0} \cos \left(72^{\circ}\right)=\sigma \mathbf{b}_{0} \quad\left[\right.$ whereCT $=\tau^{-1}=(\sqrt{5}-1) /$
2] and $\mathbf{d}_{1}+\mathbf{d}_{4}=-\tau \mathbf{d}_{0}$, a linear array of $\mathrm{BO}$ and $\mathrm{A}=\mathrm{B}$ $+\mathbf{B}_{4}$ dislocations, with the density of type $\mathrm{BO}$ chosen to be $T$ times that of type A, has a total $b$ component (phonon part) proportional to $L$ and a total $d$ component (phason part) that vanishes as $L^{-1}$.

There are two natural choices for such an array: (1) a periodic array of dislocations of types $\mathrm{BO}$, with spacing $D$ between successive dislocations and, superimposed on this array, another periodic array of dislocations of type A, with spacing $r D$ between successive dislocations; (2) an array with equal spacings between successive dislocations, but a quasiperiodic sequence of dislocations of types $\mathrm{BO}$ and $\mathrm{A}$, for example, the Fibonacci sequence BoABoBoA. . . obtained by starting with the symbol BO and using the recursion scheme $\mathbf{B}_{\mathbf{0}} \rightarrow \mathrm{BoA}, \mathbf{A} \rightarrow \mathrm{BO}$ an infinite number of times. Note that dislocations can come arbitrarily close to each other in the first array but not in the second. ${ }^{9}$ This makes the latter more appealing than the former on physical grounds; however, calculations of the grain-boundary energy become analytically tractable if we use the first array (see below). We do not expect these two different arrays to yield qualitatively different elastic energies.

In Fig. 1 we show a density-wave picture ${ }^{7(b)}$ of a symmetric tilt boundary in a two-dimensional, pentagonal quasicrystal, obtained using array type (2). Eight $\mathbf{B}_{0}$ and five A dislocations are placed along the $x$ axis in a Fibonacci sequence with a spacing $D=2 b$ between successive dislocations. Thus the mean tilt angle is 6 $=\tan ^{-1}[(8+5 \sigma) / 24]=24.8^{\circ}$. The density at $\mathbf{r}=(x, y)$ is $\mathrm{p}(\mathrm{r})=\Sigma_{n} \exp \left(i \mathbf{G}_{n} \cdot \mathbf{r}+i \phi_{n}\right)$, where

$$
\mathbf{G}_{n}=(\cos (2 \pi n / 5), \sin (2 \pi n / 5)), n=0, \ldots, 4,
$$

$\boldsymbol{\phi}_{n}=\mathbf{G}_{n} \cdot \mathbf{u}^{T}+\mathbf{G}_{\langle 3 n\rangle_{s}} \cdot \mathbf{w}^{T}$, and the total phonon and phason fields $\mathbf{u}^{T}$ and $\mathbf{w}^{T}$ are, respectively, the sums (obtained numerically) of the $u$ and $w$ fields of individual dislocations. These dislocation fields have been calculated by De and Pelcovits. ${ }^{8}$ For purposes of simplicity, hereafter we set $K_{2}=K_{3}=0$ [see Eq. (1)]. Then, for one dislocation,

$$
\begin{aligned}
& u_{x}(\mathbf{r})=\frac{b_{x}}{2 \pi}\left[\tan ^{-1}\left(\frac{y}{x}\right)+\alpha_{1} \frac{x y}{r^{2}}\right] \\
& +\frac{b_{y}}{2 \pi}\left[\alpha_{2} \ln \left(\frac{r}{a}\right)-\alpha_{1} \frac{x^{2}}{r^{2}}\right], \\
& u_{y}(\mathbf{r})=\frac{b_{x}}{2 \pi}\left[-\alpha_{2} \ln \left(\frac{r}{a}\right)+\alpha_{1} \frac{y^{2}}{r^{2}}\right] \\
& +\frac{b_{y}}{2 \pi}\left[\tan ^{-1}\left(\frac{y}{x}\right)-\alpha_{1} \frac{x y}{r^{2}}\right], \\
& w_{x}(\mathbf{r})=\frac{d_{x}}{2 \pi} \tan ^{-1}\left(\frac{y}{x}\right) \text {, } \\
& w_{y}(\mathbf{r})=\frac{d_{y}}{2 \pi} \tan ^{-1}\left(\frac{y}{x}\right) \text {, }
\end{aligned}
$$


$\mathbf{r}=\mid \mathbf{r}, \alpha_{1}=(\mu+\lambda) /(2 \mu+\lambda)$, and $\alpha_{2} \equiv 1-\alpha_{1}$. By using Eqs. (3) we calculate $p(r)$. We then obtain Fig. 1 by darkening areas where $p(r)>-0.5$. This figure shows clearly the tilt associated with the grain boundary that we have constructed.

We calculate the stress fields and energy of the grain boundary we have constructed by generalizing Read and Shockley's method for crystals. ${ }^{4}$ The phonon and phason stress fields cry and $\boldsymbol{P}_{i j}$ are determined in terms of the corresponding strain fields in the standard way by the relations cry $=\delta E_{\mathrm{el}} / \delta u_{i j}$ and $P_{i j}=\delta E_{\mathrm{el}} / \delta w_{i j}$, with $E_{\mathrm{el}}$ given by (1). For the grain boundary, we calculate cry and $P_{i j}$ by summing the contributions from each dislocation in the array. The contribution from the vth dislocation, located at $\mathbf{r}^{(v)}=\left(x^{(v)}, y^{(v)}\right)$, and with Burgers vector $\left(0, b^{(v)}, 0, d^{(v)}\right)$ is easily shown to be

$$
\begin{aligned}
& \sigma_{x y}^{(v)}(\mathbf{r})=\frac{\mu \alpha_{1} b^{(v)}}{\pi} y y_{\left[\left(x-x^{(v)}\right)^{2}+\left(y-y^{(v)}\right)^{2}\right]^{2}}, \\
& P_{x y}^{(v)}(\mathrm{r})=\frac{-K_{1} d^{(v)}}{2 \pi} \frac{y-y^{(v)}}{\left(x-x^{(v)}\right)^{2}+\left(y-y^{(v)}\right)^{2}} .
\end{aligned}
$$

For an array of type (1), with the two types of dislocations BO and A located, say, at $x=\left(m+\frac{\dot{-}}{2}\right) D$ and $x$ $=\left(m+\frac{1}{2}\right) \tau D$, respectively, with $m$ any integer, the sum of cry and $\boldsymbol{P}_{i j}$ can be done exactly using standard methods of complex analysis. ${ }^{10}$

Given cry and $\boldsymbol{P}_{i j}$, a straightforward generalization of Read and Shockley's approach shows that the elastic energy per unit length of our grain boundary can be expressed as the sum of contributions from each dislocation:

$E=\lim _{L \rightarrow \infty}\left(-\frac{1}{2 L} \sum_{v} \int_{\Gamma_{\nu}} d l\left[\sigma_{x y}(\mathbf{r}) b^{(v)}+P_{x y}(\mathbf{r}) d^{(v)}\right]\right)$,

where the integral is evaluated along the slip plane $\Gamma_{v}$ of the vth dislocation. For rational, small-angle grain boundaries in crystals, the integral is the same for each dislocation in the array, and hence the sum is trivial. However, each dislocation in a small-angle grain boundary in a quasicrystal (and in an irrational boundary in a crystal) experiences a different stress field and, therefore, contributes a different amount to the total grain-boundary energy. Thus, the total grain-boundary energy per dislocation is the average of the contributions of all dislocations. Since the set of distances between dislocations of types BO and A is uniformly dense, we approximate this average as follows: The stress-field environment of every Bo-type dislocation can be simulated by focusing on a single Bo-type dislocation (e.g., at $x=-D / 2$ ) and successively displacing the sublattice formed by the Atype dislocations along the $x$ axis through distances belonging to this dense set. Hence we replace the sum by an integral over the continuum of distances $[-\tau D / 2, \tau D / 2]_{\text {of }}$ A-type dislocations from the Bo-type dislocation sustained at $x=-D / 2$. A similar calcula- tion is also done for A-type dislocations.

We find that the energy per unit length of a symmetric grain boundary with tilt angle 9 is

$$
E=\left(A_{u} E_{u}+A_{w} E_{w}\right) \theta-\left(E_{u}+E_{w}\right) \theta \ln \theta,
$$

where

$$
\begin{aligned}
& A_{u}=1+\ln \left(\frac{2-\sigma}{\pi \xi}\right)+\left(\frac{2 \sigma+1}{2 \sigma}\right) \pi^{2} \ln 2, \\
& A_{w}-\ln \left(\frac{2-\sigma}{2 \sqrt{2} \pi \xi}\right), \quad E_{u}-\frac{\mu \alpha_{1} b}{2 \pi}\left(\frac{2 \sigma}{2-\mathrm{cr}}\right), \\
& E_{w}=\frac{K_{1} b}{4 \pi}\left(\frac{2+\mathrm{CT}}{2-\sigma}\right) .
\end{aligned}
$$

Here the core size is assumed, reasonably, to be $\xi$ times $\max (b, d)$, where $\xi$ is a dimensionless number of order unity.

In crystals small-angle grain boundaries are arrays of elementary dislocations since this minimizes the energy. However, we find that in a quasicrystal the dislocations in a grain boundary may be elementary or composite. To see this, note that we can also construct the above grain boundary by using composite dislocations with Burgers vectors $\mathbf{A}^{(N)}=\left(\tau^{N-1} \mathbf{b}_{0},-\sigma^{N-1} \mathbf{d}_{0}\right), \quad \mathbf{B}^{(N)}$ $=\left(\tau^{N} \mathbf{b}_{0}, \sigma^{N} \mathbf{d}_{0}\right)$, obtained as the $N$ th iterate of the recursive scheme $\mathbf{B}_{0} \rightarrow \mathrm{Bo}+\mathrm{A}, \mathbf{A} \rightarrow \mathrm{BO}$, and $D \rightarrow \tau D$. The $u$ parts of these Burgers vectors are larger and the $w$ parts are smaller than those of the original array. The energy of this grain boundary is

$$
E^{(N)}=\tau^{N} \theta\left[A_{u} E_{u}-E_{u} \ln \theta\right]+\sigma^{3 N} \theta\left[A_{w} E_{w}-E_{w} \ln \theta\right] .
$$

Minimizing $E^{(N)}$ (at fixed 9) with respect to $N$, we find that the optimal array has Burgers vectors with magnitudes

$$
|\mathbf{b}| \sim\left(K_{1} / \mu\right)^{1 / 4}, \quad|\mathbf{d}| \sim\left(K_{1} / \mu\right)^{-1 / 4} .
$$

Thus, no clumping of dislocations in the grain boundary will occur unless the phason elastic constant $K_{1}$ is sufficiently large. For substantial clumping $\left(K_{1} / \mu\right)^{1 / 4}$ must be much larger than 1 .

We emphasize that we have obtained the energy of a specific array [type (1)] of dislocations that leads to an acceptable grain boundary. We have not carried out a global energy minimization that would yield the thermodynamically stable grain boundary. Of course, the qualitative form of Eq. (5) and our results on clumping should not be affected by such a global minimization.

Note that each dislocation in our grain boundary produces appreciable fields only within a region around it whose linear size is of the order of the dislocation spacing $D$. Our treatment therefore assumes an equilibrium elastic theory description only out to length scales of order $D$. For $\theta=3^{\circ}$ and $u$ Burgers vector $=5 \mathrm{~A}, D=100$ A. If we assume ${ }^{11}$ phason relaxation proceeds at the 
same rate as vacancy diffusion $\left(10{ }^{12} \mathrm{~cm}^{2} / \mathrm{sec}\right)$, then phasons should have equilibrated on length scales of order $D$ in a few seconds. Our description is therefore justified.

We expect qualitatively similar results for threedimensional quasicrystals. We will publish the details of our calculations elsewhere along with straightforward generalizations of our calculations to asymmetric boundaries, to the case of three-dimensional ${ }^{12}$ and decagonal $^{12}$ quasicrystals, and to incommensurate crystals. ${ }^{13}$ The equilibrium shapes of grain inclusions in quasicrystals will follow simply from our results. ${ }^{14}$

After this work was completed, we came to know of work $^{15}$ by Rivier and Lawrence, Gratias and Thalal, and Sutton where it is pointed out that irrational boundaries in periodic crystals are quasiperiodic structures.

We hope our results will lead to careful experiments on grain boundaries in quasicrystals. Detailed microscopy should reveal the quasiperiodic arrangement of dislocations in the boundary, which is the most important qualitative result of our theory. Also, the angle dependence of the grain-boundary energy should be measurable. ${ }^{4}$ Lastly, measurements of the elastic constants of quasicrystals would make it possible to test our ideas on clumping, although detailed comparison will only be possible after calculations have been done for a three-dimensional system.

We would like to thank the University Grants Commission and the Department of Science and Technology (India) for support, John Toner for extremely useful discussions, and Yashowanto Mohapatra and Madan Rao for help with graphics. S.R. also acknowledges the hospitality of the Institute for Theoretical Physics at the University of California, at Santa Barbara, where part of this work was done.

${ }^{1}$ C. Kittel, Introduction to Solid State Physics (Wiley, New York, 1976), pp. 574-576.

${ }^{2}$ C. H. Chen, J. P. Remeika, G. P. Espinosa, and A. S. Cooper, Phys. Rev. B 35, 7737 (1987).

${ }^{3}$ F. C. Frank, in Report of the Symposium on the Plastic Deformation of Crystalline Solids (Carnegie Institute of Technology, Pittsburgh, 1950), p. 150.

${ }^{4}$ N. T. Read and W. Shockley, Phys. Rev. 78, 275 (1950); J. P. Hirth and J. Lothe, Theory of Dislocations (Wiley Interscience, New York, 1982), Chap. 19.

${ }_{5}^{5} \mathrm{~S}$. Ramaswamy and J. Toner (to be published).
${ }^{6}$ We do not consider the effect of elastic screening that modifies the results of Read and Shockley (Ref. 4) for asymmetric tilt boundaries in crystals; such effects have been considered recently for crystals by D. P. DiVincenzo and C. Rottman, Phys. Rev. B 37, 5242 (1988).

${ }^{7}$ (a) P. A. Kalugin, A. Yu. Kitaev, and L. C. Levitov, J. Phys. Lett. 46, L601 (1985); P. A. Kalugin, A. Yu. Kitaev, and L. C. Levitov, Pis'ma Zh. Eksp. Teor. Fiz. 41, 119 (1985) [JETP Lett. 41, 145 (1989)]; P. Bak, Phys. Rev. Lett. 54, 1517 (1985). (b) D. Levine, T. C. Lubensky, S. Ostlund, S. Ramaswamy, P. J. Steinhardt, and J. Toner, Phys. Rev. Lett. 54, 1520 (1985). These authors have an additional term in the elastic energy that couples $u$ and $w$ fields; such a term is present only for chiral, pentagonal quasicrystals which we do not consider.

${ }^{8}$ P. De and R. A. Pelcovits, Phys. Rev. B 35, 8609 (1987).

${ }^{9}$ Dislocations come arbitrarily close to each other in the Read-Shockley construction (Ref. 4) of an asymmetric tilt boundary in a square lattice with tilt angle 0 and oriented at an angle $\phi$ to the $x$ axis with $\tan \phi$ irrational. Such a boundary consists of two types of dislocations, say $A$ and $B$, with Burgers vectors ax and ay, respectively (a is the lattice spacing). If $D A$ $\left(D_{B}\right)$ is the $A-A(B-B)$ distance, then $\tan \phi=D_{B} / D_{A}$. Clearly, if $\tan \phi$ is irrational, dislocations of type $A$ must come arbitrarily close to dislocations of type $B$ at some points. Indeed it is easy to see that a symmetric tilt boundary in a quasicrystal must be the projection of an irrational tilt boundary perpendicular to the projection plane in a higher-dimensional crystal. For the projection of crystals to lower-dimensional quasicrystals see, e.g., V. Elser, Acta Crystallogr., Sect. A 42, 36 (1986); P. J. Steinhardt and S. Ostlund, The Physics of Quasicrystals (World Scientific, Singapore, 1987).

${ }^{10} \mathrm{G}$. F. Carrier, M. Krook, and C. E. Pearson, Functions of a Complex Variable (McGraw-Hill, New York, 1966), pp. 95-101.

"T. C. Lubensky, S. Ramaswamy, and J. Toner, Phys. Rev. B 32, 7444 (1985).

${ }^{12}$ For recent reviews see C. Henley, Comments Condens. Matter Phys. 13, 59 (1987); Steinhardt and Ostlund, Ref. 9.

${ }^{13}$ See, e.g., Incommensurate Phases in Dielectrics, edited by R. Blinc and A. P. Levanyuk (North-Holland, Amsterdam, 1986).

${ }^{14}$ Y. He, C. Jayaprakash, and C. Rottman, Phys. Rev. B 32, 12 (1985).

${ }^{15}$ N. Rivier, J. Phys. (Paris) Colloq. 47, Suppl. No. 7, C3-299 (1986); N. Rivier and A. J. A. Lawrence, Physica (to be published); in Quasicrystalline Materials, Proceedings of the Institut Laue Langevin-CODEST Conference on Quasicrystalline Materials, Genoble, 1988, edited by Chr. Janot (World Scientific, Singapore, 1988); A. Sutton, Acta Metall. 36, 1291 (1988); D. Gratias and A. Thalal, Philos. Mag. Lett. 57, 63 (1988). 